\title{
ALL THAT GLITTERS IS NOT GOLD: APPROACHES TO LABELING FOR EM
}

\author{
R.M. Albrecht and D.A. Meyer \\ Department of Animal Sciences, University of Wisconsin, Madison, WI 53706
}

A large number of natural and synthetic species ranging from virus particles to small polymer beads have been employed over the years as labels in electron microscopic applications. Perhaps the most useful and versatile of these labels are the colloidal heavy metals.[1] The colloidal labels can be synthesized as round regular spheres in sizes from 1 to 150 nanometers in diameter and thus can be used where molecular and sub-molecular ranges of spatial resolution are required. They are electron "dense" and good emitters of secondary and backscattered electrons and hence are readily detectable in both scanning electron microscopic imaging systems and transmission electron imaging systems. Larger particles, $10 \mathrm{~nm}$ and above, can be identified via their shape in force based imaging systems. Each particle is basically a small sphere containing a number of atoms of the particular element in a relatively small volume. This improves detectability over less dense metal-organic complexes or proteins that are bound to specific elements such as boron or iron. In the latter case the metal density is relatively low and substantial numbers of the labels are often required for detection.

The bulk concentration of the colloidal particles in a tissue volume can be determined at very high sensitivity via Instrumental Neutron Activation Analysis, INAA, with minimal preparation and without significant specimen damage.[2] INAA analysis can be performed prior to or after imaging. INAA is very valuable in determining bulk concentrations of label to levels that would be difficult to detect by sectioning and counting due to insufficient particle numbers. INAA can be used with gold and other colloids to further improve the quantitative aspects of multiple labeling for EM. Label density in bulk, unsectioned tissue can be determined using INAA This permits a prediction of the number of labels in, for example, thin sections or whole mounts prepared from the labeled tissue. If particle numbers, as measured by INAA of bulk tissue, are too low or too high, labeling parameters or experimental design can be adjusted prior to tissue fixation, dehydration, embedding, sectioning, and imaging.[3]

The colloidal particles are also useful for correlative microscopic investigations since individual particles as small as $12 \mathrm{~nm}$ can be detected (but not resolved) and tracked via their inflated diffraction image using interference based light microscopy.[1] Individual particles can also serve as nuclei for further addition of silver or gold in a process that enlarges each particle to a size that is resolvable in conventional light microscopy. Relatively small numbers particles can darken or color areas they stain due to their extremely high extinction coefficients compared to other dyes.

For use in labeling, colloidal particles can be readily conjugated, principally via strong hydrophobic interactions, to a variety of biologically active molecular species. The conjugates are stable and very little loss of ligand/antibody activity or specificity is encountered. A thin shell of bound ligand or antibody surrounds larger particles while the smaller particles intercalate within the structure of individual ligand or antibody molecule. Very small particles in the 2 to 5 nanometer range can be conjugated to active fragments of ligands or antibodies to produce labels with dimensions that readily permit identification and localization at sub-molecular levels of spatial resolution and with 
minimal steric interference. One small colloidal particle, conjugated to an active ligand fragment or a single Fab of an antibody molecule, results in labels having a single valence and hence one particle represents one binding site or epitope. This is advantageous for quantitative studies.

One of the critical factors in understanding cellular function is knowledge of the complex and dynamic cellular architecture and the interactions of individual molecular species within that architecture. Co-localization studies employing simultaneous multiple labels are often an extremely effective way in which questions of this type can be addressed. Photon based imaging systems use fluorescent dyes having different excitation and emission wavelengths. For studies at molecular and sub-molecular levels of resolution the standard approach is to use colloidal gold particles of different sizes. Unfortunately, to ensure that overlap in particle size is not a problem, generally only one or two particle sizes within the range that provide the desired level of resolution can be employed at once. Attendant steric problems arise because of the substantially greater mass of the larger particle. Substantial differences in ease of detection of the two particles also occur due to the larger size and greater mass of the larger gold particle. Therefore accurate localization/co-localization of multiple molecular species requires a labeling system that uses small particles of nearly identical size that can be conjugated to different identifier molecules (antibodies or ligands), or active fragments of antibodies or ligands. The different small conjugates permit simultaneous labeling of multiple epitopes or binding sites. However, the different particles must be easily and reproducibly identified from one another. We are pursuing two approaches to achieving this end.[4,5,6] The first is the synthesis of uniformly sized colloidal metal particles of differing geometry.[4] Unique shapes (spheres, asterisks, geometric, irregular) based on differences in particle nucleation and growth parameters, can be detected using standard TEM and SEM (and, within limitations, by Force Microscopy) This is assuming the shape variation is within the resolving limits of the particular instrumentation available. The second approach is the synthesis of uniformly sized colloidal metal particles of differing elemental composition such as $\mathrm{Au}, \mathrm{Ag}, \mathrm{Pd}, \mathrm{Pt}, \mathrm{Ru}, \mathrm{Rh}$.[5] Energy Filtering Electron Microscopy, EF-TEM, is used to identify and differentiate particles that are of the same size but of different elemental composition.[5,6] EF-TEM relies on the electron energy loss spectra, EELS, of different elements to selectively image materials. Colloidal particles of similar size but of differing composition will produce different, generally non-overlapping, energy losses in the beam electrons. Thus a typical EM image demonstrating ultrastructural features can be collected using zero loss electrons (zero loss also minimizes chromatic aberration seen with conventional EM imaging). Subsequently, labels of similar sizes but composed of different colloidal metals can be differentiated via EF-TEM imaging. Both shape and composition can be used in concert to differentiate particles and permit simultaneous identification and co-localization of multiple molecular species at molecular levels of resolution for in vitro and in vivo applications.

References

[1] R.M. Albrecht et. al., Immunochemistry, A Practical Approach, Oxford Univ. Press. Oxford (1993)151.

[2] J.F. Hillyer and R.M. Albrecht, Microsc. and Microanal. 4 (1999) 481.

[3] J.F. Hillyer and R.M. Albrecht, J. Pharm. Sci. 90 (2001) 1927.

[4] R.M. Albrecht and D.A. Meyer, Microsc. and Microanal.6 (Suppl. 2) (2000) 318.

[5] D.A. Meyer and R.M. Albrecht, Microsc. and Microanal. 6 (Suppl. 2) (2000) 322.

[6] D.A. Meyer and R.M. Albrecht, Microsc. and Microanal. 7 (Suppl. 2) (2001) 1032. 